\title{
Fast cell selection with efficient active set management in OFDMA networks with femtocells
}

\author{
Zdenek Becvar ${ }^{1 *}$, Pierre Roux ${ }^{2}$ and Pavel Mach ${ }^{1}$
}

\begin{abstract}
In 4G wireless networks, only hard handover is defined to support users' mobility. However, dense deployment of femtocells leads to significant rise in amount of initiated handovers. Thus, inevitable decrease in quality of service (QoS) experienced by users is observed. In this article, we investigate possible introduction of fast cell selection (FCS) to OFDMA-based network with femtocells. The goal is to minimize negative impact of user's mobility on user's QoS and to take an advantage of macro diversity. First, we define necessary enhancements in current LTE standards to facilitate implementation of FCS to OFDMA networks. Afterward, impact of FCS on network performance is evaluated. Second, we propose novel algorithm for active set management, which considers specifics of femtocells. As simulation results demonstrate, even FCS with conventional active set management is profitable for the networks performance. Nevertheless, the proposed innovation of active set management procedure further improves efficiency of FCS comparing to existing schemes. We also present the most common content of active set observed from simulations if novel algorithm is exploited.
\end{abstract}

\section{Introduction}

By deployment of open or hybrid access femtocells with low coverage radius into OFDMA wireless networks, the number of initiated handovers can rise significantly. This results in decrease of quality of service (QoS) for user equipment (UE). The lowering of QoS is caused by short interruption introduced by each handover procedure [1]. The reduction of amount of performed handovers can be achieved by conventional techniques, such as hysteresis or time-to-trigger timer. These techniques can be implemented also in networks with femtocells as presented, e.g., in [2]. Nevertheless, a gain introduced by open access femtocells [3] drops rapidly by using conventional techniques eliminating redundant handovers [4].

A solution for this problem can consist in soft handover or fast cell selection (FCS). The major difference between both solutions lies in a way of transmission between a UE and neighboring cells included in its active set. In case of soft handover, all cells in active set transmit data simultaneously and the receiver combines all data in macro diversity manner. The FCS offers means

\footnotetext{
* Correspondence: zdenek.becvar@fel.cvut.cz

'Department of Telecommunication Engineering, Czech Technical University in Prague, Technicka 2, 16627, Prague, Czech Republic

Full list of author information is available at the end of the article
}

for the terminal to decide which cell in its active set is really going to send data in the next transmission time interval (TTI). To that end, FCS selects and updates the best cell for transmission at each transmission interval. Thus, the same data are not sent multiple as in case of soft handover technique.

Soft handover is known as a CDMA-specific technique, which cannot be ported into OFDMA-based systems unless particular algorithms are used at the physical layer in order to achieve cooperation among base stations, as described in [5]. The FCS is actually a technique derived from CDMA soft handover. Consequently, its implementation into OFDMA-based system requires specific modification at physical layer as well.

In this article, we focus on FCS since it does not implicate any advanced requirements, such as support of macro diversity combining, on UEs. The FCS can be beneficial for indoor as well as outdoor UEs under the coverage of several femto access points (FAPs) or combined coverage of macro base stations (MBSs) and several FAPs. Nevertheless, several problems must be solved for efficient exploitation of FCS in 4G networks with FAPs. First, if a UE consumes significant part of resources at the FAP (e.g., due to low signal level), the same resources (at the same frequencies and in the same time intervals) should not be used by other active set 
members in case of the conventional FCS or FCS with frequency muting. Thus, it could limit the radio capacity of the MBS. Second, if FCS is enabled, user's data intended for each UE must be routed into all cells in its active set. Due to the limitation of FAPs backbone, inclusion of a FAP in active sets should consider also the backbone capacity of individual cell especially if the FAP is inactive in transmission to the UE. This problem does not occur in scenario with conventional MBSs only as the cellular backbone network usually provides very high capacity. Nevertheless, the backbone of femtocells is of notably lower quality (in most cases, the FAPs are connected to operator network via a DSL). Last, FAP's backbone is also of a variable quality. If two FAPs are in the active set of a UE, we can assume that those belong to the same operator (otherwise, FCS would not be possible as user is usually subscribed only at one operator). If an MBS and one or several FAPs are included, we have to ensure that data will be ready at the same time at the FAPs and the MBS. In praxis, it means to increase packet delay to the maximum delivery delay observed among all cells in active set.

The contribution of this article is as follows. First, we propose new method to adapt FCS principles to OFDMA system with the femtocells. Subsequently, evaluation of FCS performance in 4G OFDMA networks with femtocells is carried out to demonstrate its efficiency. Second, novel algorithm selecting suitable candidates to be included/deleted to/from active set is presented. The performance of the proposed algorithm is compared to the conventional FCS and to the most promising competitive algorithm. We also discuss size and content of active set for potential further exploitation by algorithms for cooperative communications.

The rest of the article is organized as follows. The next section gives an overview on related work in area of FCS and active set management. "FCS in OFDMA networks with femtocells" section describes modifications required for implementation of FCS to OFDMA networks with femtocells. Further, the same section shows improvement in network performance if FCS is used. "Active set management" section proposes an innovative algorithm for active set management, comparison of its performance with competitive schemes, and discussion of backbone overhead introduced by this algorithm. The last section summarizes major conclusions and future work plans.

\section{Related work}

The FCS has been introduced in 3GPP Release 99 as the SSDT (Site Selection Diversity Transmission) feature (see [6,7]). In 3GPP Release 99, FCS strongly relies on the use of CDMA while only MBSs are considered. Therefore, we define modifications needed for utilization of FCS in OFDMA networks with femtocells in this article.

An active set must be set up for each UE if FCS is enabled. This active set is continuously updated by means of either inclusion or deletion of cells that can potentially either improve or deteriorate performance, respectively. The authors of [8] compare fractional frequency reuse in a single cell transmission scenario with FCS enhanced by adaptive MIMO mode selection in combination with interference avoidance technique. The investigation is done for active set encompassing two and three MBSs. The active set is updated according to the signal level received from neighboring BSs. The consideration of relation among signal levels of neighboring cells is the conventional approach for FCS.

The authors of $[9,10]$ propose new metric, denoted as interference to other interferences plus noise ratio (IINR), for active set management. In comparison to the conventional SINR, the IINR does not take signal level of the serving BS into account. The measurement of IINR requires no transmission on resource elements (REs) that are occupied by reference signals of neighboring BSs. The IINR introduces gain in spectral efficiency for cell edge users and simultaneously it reduces amount of candidate cells reported by the UE. This way, load in uplink is reduced while downlink is unaffected.

The authors of [11] propose frequency muting for FCS. The muting is applied to the second strongest cell according to the UE's measurement. As the results show, this approach can introduce roughly $10 \%$ gain in throughput of cell-edge users comparing to single cell transmission. Further gain of additional $10 \%$ can be introduced by joint processing. However, this is obtained at the cost of much higher complexity. Further extension of the muting idea is presented in [12]. The authors propose adaptive muting based on capacity calculation and power allocation based on muting mode selection. The muting is applied to all resource blocks (RBs) to avoid power wasting. Hence, transmitting power at some RBs is lowered while the power of some RBs is boosted. Nevertheless, the overall transmission power is kept as in the conventional case. The muting mode is considered only if UE's capacity is at least double comparing to non-muting mode. The results show improvement in throughput by roughly $5.5 \%$ comparing to the single cell transmission.

Analyzing an impact on throughput if a new cell is included in an active set is presented also in [13]. The authors compare the performance in case when the candidate cell would be included with the case when it is not. If the gain by inclusion of the cell exceeds predefined threshold, the update of active set is performed.

The FCS introduces a gain in throughput especially at the cell edges where the interference is not marginal as 
shown, for example, in [14,15]. All above-mentioned articles investigate FCS in scenario with macrocells only. However, while FAPs are considered, the users are located at cell edges more often than in case of conventional MBSs.

Comparing to listed related work on active set management, our proposal differs in several aspects. First, we consider deployment of FAPs and its related backbone problems. As already mentioned, large amount of radio resources of an MBS could be wasted if the MBS would be included in active set together with a FAP with weak signal. Therefore, comparing to $[11,12]$, our proposal is based on evaluation of the impact of active set enhancement on amount of consumed radio resources of the MBS. Nevertheless, the frequency muting according to [12] is considered in our proposal. Contrary to [12], the muting is not limited only to the second strongest cell, but it is applied to all cells in active set if profitable. Further, limitation of FAP's backbone capacity and delay are considered in our proposal. As FAPs are supposed to be connected via DSL connection, the backbone capacity is significantly lower than the capacity of MBSs backbone. Thus, each inclusion of a FAP into the active set should take the backbone limitation into account. In addition, FAP's backbone delay is a new parameter considered when updating active set in our proposal since this delay is typically higher than that of MBSs backbone.

\section{FCS in OFDMA networks with femtocells}

This section describes the proposal of modification of the conventional CDMA FCS to be applicable in OFDMA-based LTE networks with femtocells. Further, evaluation of OFDMA FCS is performed to show its efficiency even in this kind of networks.

\section{From CDMA FCS to OFDMA FCS}

The first requirement that the OFDMA system has to fulfill for FCS is a time synchronization among cells in the network. Without synchronization, only classical handover is possible, where the UE needs to resynchronize itself on the target cell after each handover. Synchronizing the system allows to see FCS as a specific case of joint scheduling, where the cells in active set collaborate in such a way that only the best cell in the set can schedule data toward the UE at each TTI. In case of TDD, time synchronization of the FAP could typically be derived from the umbrella MBS.

Then, each cell in the active set should receive the integral data to be scheduled toward the UE. This principle introduces redundancy since all non-transmitting FAPs will have to discard data received from the serving gateway $(\mathrm{S}-\mathrm{GW})$. However, it allows reaching high rates of cell switching without flooding the mobility management entity with handover events.

Since OFDMA systems, such as LTE, do not address the notion of soft handover with active set of cells serving a given UE, a solution is needed to allow several FAPs or MBSs to participate in an active set. Once a radio bearer is established for a UE with one cell in the data path, then it should be possible to add and remove additional cell contributors. This introduces the notion of a "serving" cell in the active set, which assumes a particular role, as opposed to a simple contributor cells. The standard handover procedure is still applied whenever the serving cell in the active set is shifted from a current one to a target one. However, no interruption is introduced in this case. Figure 1 illustrates the needed modifications for implementing FCS into LTE architecture with FAPs. This figure shows an active set made of one macrocell and two femtocells, with one femtocell playing the serving role. The figure assumes a specific time slot when one cell is selected to send data to the UE (represented by the solid arrow from this cell to the UE). Dashed lines represent channel from contributing cells that can be used for transmission in one of following frames. The PDN-GW refers to the packet data network gateway, while the S-GW refers to the serving gateway.

The control procedure should be defined in order to allow adding or removing contributing cells to or from the active set (algorithm for adding/removing cells to/ from active set is elaborated later in this article). When a contributing cell is added to the active set, then the $\mathrm{S}$ -

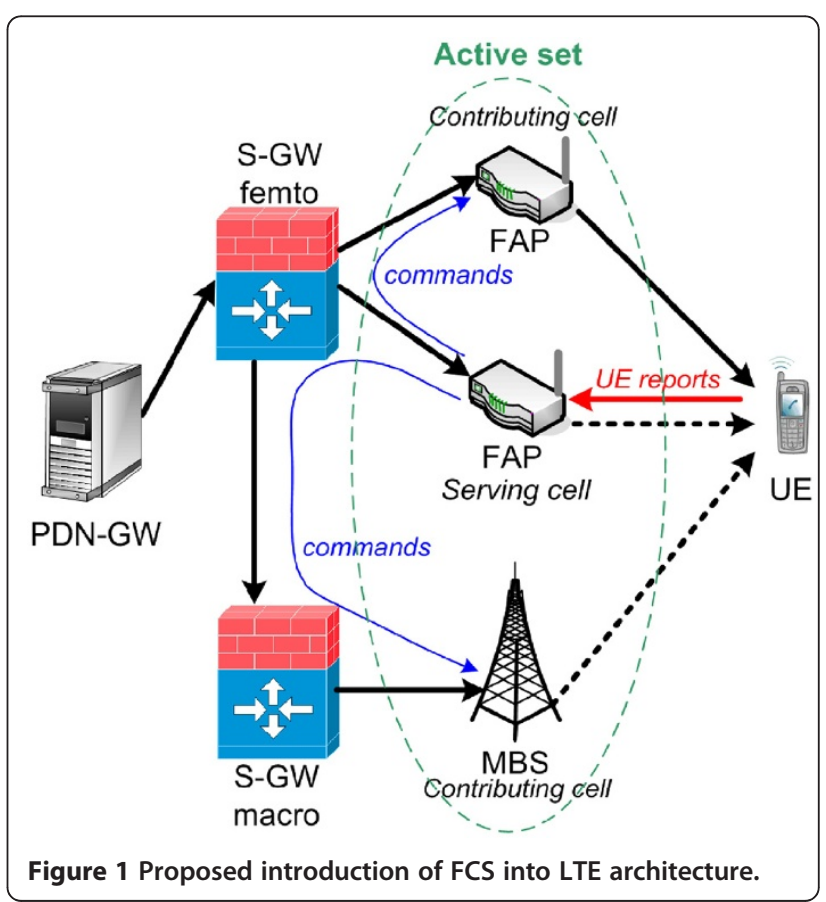


GW should be notified and it should duplicate packets toward the new cell in the downlink. Similar mechanisms could be proposed for the uplink so that contributing cells may have a role in both uplink and downlink. This article focuses only on downlink aspects.

The serving cell should be in charge of adding or removing additional contributor cells in the active set. When the serving cell is shifted from a source one to a target one, the set of contributing cells should be communicated from the current serving cell to the target serving cell as a part of the UE context. Once a successful handover is achieved for a UE, then the target cell is free to either maintain or modify the set of cell contributors that were used in the former serving cell.

A solution should also be proposed in order to let contributing cells know if they are elected for scheduling data toward the UE for a given TTI. The solutions, which have been defined in the context of 3GPP Release 99, are CDMA specific and cannot be applied outside this context.

The most natural solution is to let the serving cell deliver this information to the contributing cells on the basis of the UE measurement reports. The serving cell should provide (and update) the list of contributing cells to the UE for this report. The serving cell should also request a report periodicity that might be as fast as once for each TTI. However, the periodicity is typically much longer in case of slow moving UEs (pedestrians) with the constraint that it should not exceed the maximum value enabled by $\operatorname{LTE}(-\mathrm{A})$, which is $160 \mathrm{~ms}$.

The nature of the measurement should also be part of the report configuration. In the simplest case, which is also the most economical one, in terms of uplink bandwidth consumption, only the index of the best cell should be sent. If a maximum active set of eight cells is considered, this would require only 3 bits.

The serving cell should exploit FCS measurement reports from the UE in order to decide, which cell in the active set will actually be in charge of scheduling data to the UE. Whenever a modification is decided in this respect, the decision should be communicated to all the involved contributing cells. This command from the serving cell to contributing cell should just include the ON/OFF Boolean value, together with a reference to the next bit of data to be sent.

Table 1 gives a summary of procedures to be added for supporting FCS in current OFDMA-based systems with FAPs.

\section{Evaluation of FCS in ODFMA networks with femtocells}

This section proposes a methodology for FCS performance evaluation together with the main simulation assumptions. Then the results of simulation focused on performance of the FCS in OFDMA networks with femtocells are presented and discussed.

\section{System model, methodology, and simulation settings}

A simulation tool has been developed in order to evaluate performance of FCS in OFDMA networks. The simulator addresses downlink communication and it assumes the MBS and the FAPs share the same frequency spectrum allocation, so that both the FAP to the MBS and the MBS to the FAP interferences are considered. All transmitters have omni-directional radiation pattern.

The femtocells with open access mode are assumed in the sense that any UE may be attached either to the MBS or to any neighboring FAP.

The size of simulated area is $2000 \mathrm{~m} \times 1000 \mathrm{~m}$. The deployment of nodes in the simulation area follows the rural scenario with an MBS in the middle of the area and with 50 houses randomly placed within the area boundaries. Individual houses have square structure, which dimension corresponds to $12 \times 12 \mathrm{~m}^{2}$. Each house is equipped with one randomly placed FAP and one UE. Additionally, 100 outdoor UEs are randomly distributed in the area.

The path attenuation from any transmitter to any receiver is made of three components. First, a static path loss is calculated according Okumara-Hata model [16] for MBS to UE communication and ITU P.1238 model [16] for FAP to UE communication. An additional wall loss equal to $10 \mathrm{~dB}$ is applied whenever the path from the FAP or the MBS crosses a house wall. Second, dynamic and flat path losses accounting for slow terminal mobility are assumed. The simulator does not apply full

Table 1 Procedures for FCS support in OFDMA-based networks with FAPs

\begin{tabular}{llll}
\hline From & To & Message purpose & Message content \\
\hline Serving cell & S-GW & Add a contributing cell & Identification of cell to be added \\
Serving cell & S-GW & Remove a contributing cell & Identification of cell to be removed \\
Serving cell & UE & $\begin{array}{l}\text { Define/update FCS } \\
\text { measurement report }\end{array}$ & $\begin{array}{l}\text { Measurement period Measurement } \\
\text { content as an index in pre-defined list }\end{array}$ \\
UE & FCS measurement report & Measured value \\
Serving cell & Serving cell & FCS command & On/Off value Identification of next bit of \\
& Contributing cell & & data to be sent (if the contributing cell is turned on)
\end{tabular}


mobility of UEs by means of trajectories; however, slow terminal mobility is assumed, as far as channel model is involved. The average amplitude of the shadowing is set to $8 \mathrm{~dB}$ when the macro cell is involved or $4 \mathrm{~dB}$ when one of the FAPs is involved [16]. The dynamic shadowing is modeled as a spectrally flat and band-limited noise with a cut off frequency equal to $3 \mathrm{~Hz}$. Last, a dynamic and frequency selective shadowing accounting for slow terminal mobility, with a Doppler frequency set to $3 \mathrm{~Hz}$, is assumed. The channel is modeled as a six-taps delay line with relative average power and delays for each tap being set according to ITU-R M.1225 [17].

The assumed channel bandwidth is set to the $20 \mathrm{MHz}$ in accordance to LTE-A release 10. The frequency resolution for selective channel simulation is equal to the channel bandwidth of one frequency RB in LTE: 180 $\mathrm{kHz}$ (i.e., $12 \times 15 \mathrm{kHz}$ ).

The simulator's time granularity is set to $1 \mathrm{~ms}$, which is the shortest duration in the LTE scheduler for assigning a radio block to a terminal. Opportunistic scheduling is assumed, in order to check FCS compatibility with the most advanced scheduling techniques. To obtain reliable results, one simulation run lasts one simulated hour.

Several simulations are run for the purpose of performance evaluation, with increasing bit-rates being sent to all UEs. Above a given bit-rate value, buffer losses are observed for the most critical UEs and become more and more frequent (with more terminals impacted) with increased bit-rates. The major simulation parameters are summarized in Table 2.

\section{Simulation results}

The goal of this section is to evaluate efficiency of FCS in OFDMA networks with femtocells. Figure 2 shows average loss ratio for outdoor (Figure 2a) and indoor (Figure 2b) users. Both figures compare system using FCS and a system, which is not. The comparison is performed from the packet loss ratio point of view. Although an opportunistic scheduling is assumed, one reference curve is introduced in the plot in order to

Table 2 Parameters and models used for evaluation

\begin{tabular}{ll}
\hline Parameters & Value \\
\hline Carrier frequency & $2.0 \mathrm{GHz}$ \\
Channel bandwidth & $20 \mathrm{MHz}$ \\
MBS/FAP transmitting power & $46 / 15 \mathrm{dBm}$ \\
Number of MBSs/FAPs & $1 / 50$ \\
Number of indoor/outdoor UEs & $50 / 100$ \\
Wall penetration loss & $10 \mathrm{~dB}$ \\
Size of simulation area & $2000 \times 1000 \mathrm{~m}$ \\
Simulation step & $1 \mathrm{~ms}$ \\
Simulation real-time & $3600 \mathrm{~s}$ \\
\hline
\end{tabular}

show performances in the absence of FCS and in case of simple round robin scheduling.

If we consider outdoor UEs and a maximum loss ratio equals, e.g., to 0.1, than we observe that FCS can bring the gain of roughly $20 \%$ in average bit rate (increase from 340 to $410 \mathrm{kbps})$. On the other view, for the same offered traffic, the values of packet losses are up to $10 \%$ lower if FCS is assumed. This improvement comes on the top of opportunistic scheduling: if neither opportunistic scheduling nor FCS would be used, the average bit rate would only reach $200 \mathrm{kbps}$ and approximately $20 \%$ of new losses are introduced comparing to the enabled FCS.

At the same time, we observe that FCS is beneficial for indoor UEs as well, though the magnitude of improvement is less significant.

As the results show, FCS is profitable also in OFDMA networks with femtocells. Nevertheless, its performance can be further improved if advanced management of active set is considered. This problem is addressed in the following section.

\section{Active set management}

Previous section demonstrates efficiency of FCS in OFDMA networks with femtocells. Now, the goal is to provide a solution for selection of active set members. Our proposal is based on the calculation of amount of consumed radio resources and on backbone quality consideration. To be more specific, the proposed algorithm compares the current amount of consumed radio resources at the MBS with the radio resources consumed if a cell would be added/removed to/from active set. In addition, the backbone limitation, in terms of limited capacity and higher delay, is introduced in the proposed active set management procedure. The proposed algorithm is assumed to be run by the serving cell (either a FAP or an MBS can take over this role). In the general and the simplest case, the MBS could run this algorithm. Nevertheless, the situation when the MBS is not reachable by the UE can occur. Therefore, we suggest to run the algorithm always by the serving cell.

\section{Notations}

In order to follow easily the explanation of the proposed algorithm, summarization of parameters used in description of the proposed active set management is presented in Table 3.

\section{Proposed algorithm for active set management}

The principle of the proposed algorithm is depicted in Figure 3. The goal of the algorithm is to include a new cell into $A^{i}$ if it meets all conditions, or to remove a cell 


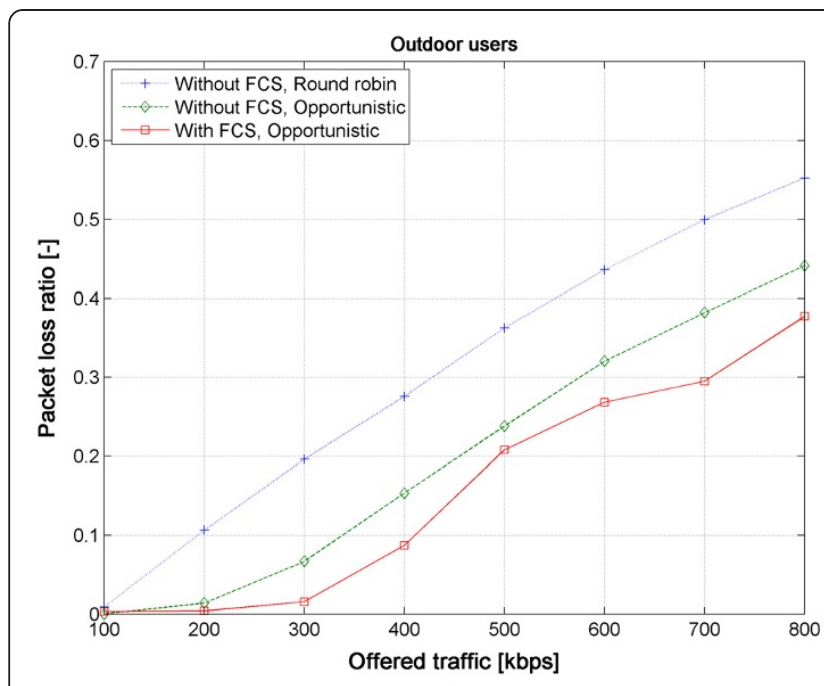

a)

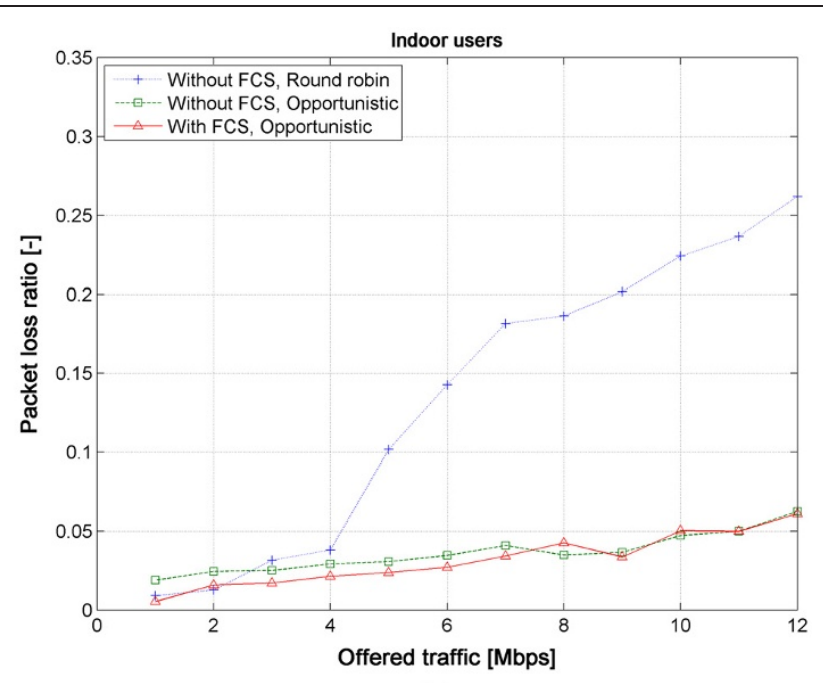

b)

Figure 2 Comparison of packet loss ratio with and without FCS.

from existing $A^{i}$ if the conditions for its addition are no longer fulfilled.

Let $U E=\left\{U E_{1}, U E_{2}, \ldots, U E_{u}\right\}$ denotes a set of $u$ users in the network and $C=\left\{C_{1}, \ldots, C_{m}, C_{m+1}, \ldots C_{m+f}\right\}$ represents the set of $k=m+f$ cells in the network, where $m$ and $f$ are the number of MBSs and FAPs, respectively. Further, $N^{i}=\left\{N_{1}^{i}, N_{2}^{i}, \ldots, N_{n c^{i}}^{i}\right\}$ represents the set of neighboring cells of $i$-th UE. Each $N^{i}$ consists of $n c^{i}$ neighboring cells. The set $A^{i}=\left\{A_{1}^{i}, A_{2}^{i}, \ldots, A_{a c^{i}}^{i}\right\}$ is composed of cells included in the active set of $i$-th UE. Note that $A^{i}$ is always a subset of $N^{i}$, i.e., $A^{i} \subseteq N^{i}$.

If $C_{j} \in N^{i}$ and $C_{j} \notin A^{i}$, then the cell can be included into the $A^{i}$ if:

$$
\alpha R_{j \in A^{i}}^{i} \leq R_{j \notin A^{i}}^{i}
$$

Note that all parameters in (1) and in any subsequent formulas are explained in Table 3.
The MBS's resources are considered in this equation rather than the FAP's resources since each FAP is supposed to serve only low amount of users comparing to the MBS. Thus, any change in an active set influences large amount of the MBS users, but only low amount of the FAPs users.

Both $R_{j \in A^{i}}^{i}$ and $R_{j \neq A^{i}}^{i}$ are derived from the reports on signal-to-noise ratio (SNR) measured by the $U E_{i}$ from all cells included in $N^{i}$ (see, e.g., [12]). If the SNR of all cells included in $N^{i}$ is measured, then the SINR can be determined. Then, the SINR is mapped to a modulation and coding scheme (MCS) according to, for example, [18]. Each MCS defines a modulation and a coding rate. Therefore, an amount of bits in a RE, denoted as $b_{R E}$, can be derived as a multiplication of the coding rate (cr) and amount of bits per symbol of the modulation (bps), i.e., $b_{R E}=c r \times b p s$. Knowing amount of the radio resources required by $U E_{i}$ and $b_{R E}$ of appropriate channel between the $U E_{i}$ and the

Table 3 Notation of parameters used for description of the proposed algorithm

\begin{tabular}{ll}
\hline Symbol & List of neighboring cells of $i$-th $\cup E, N^{i}=\left\{N_{1}^{i}, N_{2}^{i}, \ldots, N_{n c^{i}}^{i}\right\}$. \\
\hline$N^{i}$ & List of cells included in active set of $i$-th $\cup E, A^{i}=\left\{A_{1}^{i}, A_{2}^{i}, \ldots, A_{a c^{i}}^{i}\right\}$. \\
$A^{i}$ & Number of cells included in neighbor cell list. \\
$n c^{i}$ & Active set size, i.e., amount of cells included in $A^{i}$ of $i$-th $\cup E$ \\
$a c^{i}$ & Amount of radio resources consumed by $i$-th $\cup E$ if cell $C_{j}$ is included in $A^{i}$ and if it is not included in $A^{i}$ respectively. \\
$R_{j \in A^{i}}^{i}, R_{j \neq A^{i}}^{i}$ & Gain required for inclusion of a cell into $A^{i}$. \\
$a$ & Delay of data delivered though cell $C_{j}$, and maximum acceptable delay for the service experienced by $i$-th $\cup E$. \\
$D_{j}, D_{s}$ & Available capacity of backbone of $C_{j}$ and capacity required by i-th $\cup E$ respectively. \\
$b_{j, a v}, b^{i, r e g}$ & Throughput of $i$-th $\cup E$ if the $C_{j}$ would be added to $A^{i}$ and throughput experienced by i-th UE from the current serving cell. \\
$T_{j, j \in A^{i}}^{i}, T_{S}^{i}$ & Gain in amount of MBS's radio resources released by inclusion of cell $C_{j}$ into $A^{i}$ related to the requested capacity. \\
$\boldsymbol{K}_{j}^{i}$ &
\end{tabular}




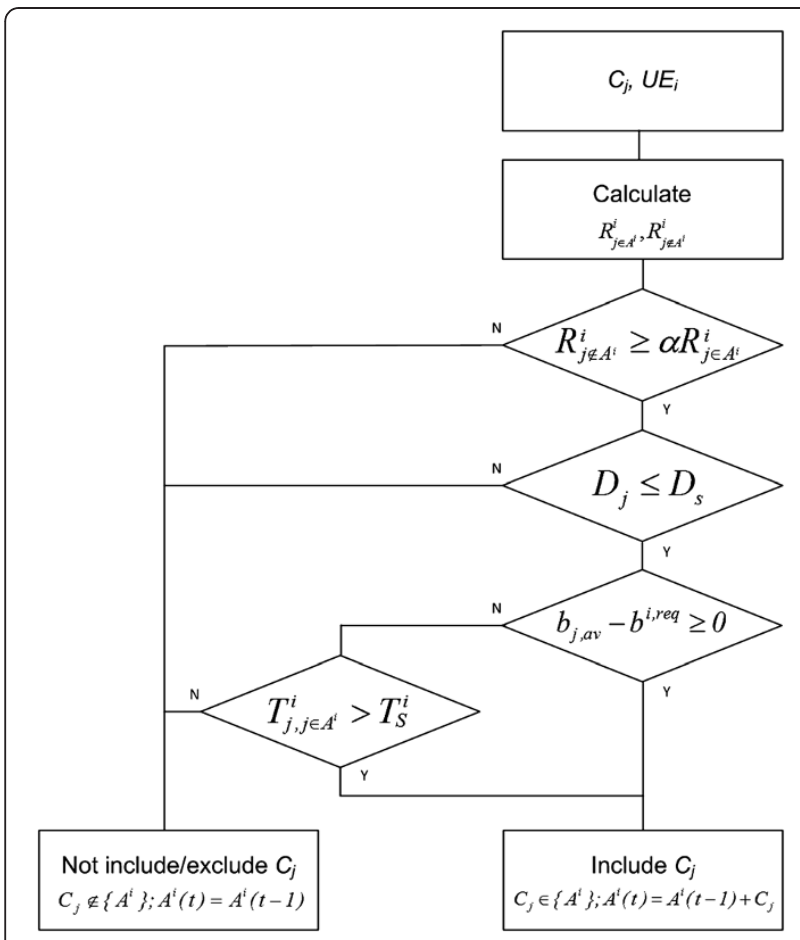

Figure 3 Proposed algorithm for active set management.

$C_{j}$, the amount of consumed resources is determined as a simple ratio of data intended to be sent by the $\mathrm{UE}_{i}\left(d_{U E}\right)$ and $b_{R E} ; R^{i}=d_{U E} / b_{R E}$. Difference in derivation of both $R_{j \in A^{i}}^{i}$ and $R_{j \notin A^{i}}^{i}$ consists in consideration of the $C_{j}$ in interference evaluation. For $R_{j \in A^{i}}^{i}$, the signal from the $C_{j}$ is not taken into account since no cell included in $A^{i}$ can transmit at the same frequencies as the serving cell. Contrary, the signal from the $C_{j}$ is included in interference for $R_{j \notin A^{i}}^{i}$.

Once the inclusion of the $C_{j}$ in $A^{i}$ is profitable from the amount of consumed MBS's radio resources point of view, the quality of the $C_{j}$ 's backbone is evaluated. A problem of packet delay due to transmission via backbones with different delays is fixed as follows. To cope with the delay, we suggest an additional condition for inclusion of a cell into $A^{i}$. This condition can be defined by the following equation:

$$
D_{j} \leq D_{s}^{i}
$$

Note that this is common problem of handover procedure. Therefore, it should be considered even in a conventional hard handover. However, the backbone of MBSs is typically provided by high-quality connection with low delay and this problem does not occur.

The backbone of MBSs is planned to be able to serve all the data transmitted via the radio interface. It means the bottleneck does not appear on the backbone of MBSs. In case of the FAPs, the situation is exactly the opposite. Since the FAPs are supposed to be connected to the network via a backbone with limited capacity, previous conditions (1) and (2) are complemented by additional one focused on the backbone capacity. The femtocell $C_{j}$ can potentially be included in $A^{i}$ only if:

$$
b_{j, a v}-b^{i, r e q} \geq 0
$$

After fulfilling (3), the $C_{j}$ is included in temporary active set $A_{\text {temp }}^{i}$. The $A_{\text {temp }}^{i}$ is composed of all cells that should be included in $A^{i}$ as those meet (1), (2), and (3). If only one UE is supposed to include the $C_{j}$ into $A^{i}$, then the temporary active set can be added to the $A^{i}$, i.e., $\left\{A^{i}\right\}=\left\{A^{i}\right\}+\left\{A_{\text {temp }}^{i}\right\}$. If more UEs would like to include $C_{j}$ in their $A^{i}$, then the backbone limit is reconsidered. The cell $C_{j}$ should be added to more active sets only if the FAP will be still able to serve all UEs as expresses the following equation:

$$
\sum_{i, i \mid j \in A_{\text {temp }}^{i}} b^{i, r e q} \leq b_{j, a v}
$$

If (4) is not fulfilled, only $A^{i}$ of selected UEs will be updated. A procedure for selecting the most appropriate UEs whose active set will be enhanced by the $C_{j}$ should be defined. For this purpose, we designate new parameter, $\kappa_{j}^{i}$. This parameter represents a ratio of gain caused by inclusion of the cell $C_{j}$ to the requested backbone capacity. It is defined by the following equation:

$$
\kappa_{j}^{i}=\frac{R_{j \notin A^{i}}^{i}-R_{j \in A^{i}}^{i}}{b^{i, r e q}}
$$

The $C_{j}$ is included only to the $A^{i}$ of UEs that leads to the highest $\kappa_{j}^{i}$. For this purpose, the $\kappa_{j}^{i}$ is reordered in descending order as follows:

$\operatorname{sort}\left(\kappa_{j}^{i} \mid j=\right.$ const $)=\left\{\max \left\{\kappa_{j}^{i}\right\}, \max \left\{\kappa_{j}^{i}-\max \left\{\kappa_{j}^{i}\right\}\right\}, \ldots\right\}$

Then the $C_{j}$ is sequentially added to the $A^{i}$ for $i=$ $1, \ldots, b_{\max }$. The $b_{\max }$ is determined as $\max (b) ; b=$ $(1, \ldots, u)$ for which the following formula is still valid:

$$
b_{j, a v}-\sum_{i=1}^{b} b^{i, r e q} \geq 0
$$

A specific situation, when (1) and (2) are fulfilled while (3) is not, can occur. In this case, a FAP can provide higher throughput even if other UEs with this FAP in active set could suffer from the inclusion of the FAP into $A^{i}$. Nevertheless, a drop in throughput of the UEs served by the FAP can be insignificant and the throughput of all of these UEs can be still above the one provided by the 
MBS. Therefore, the cell $C_{j}$ is included into $A^{i}$ even if not enough available backbone is provided by $C_{j}$. The inclusion is conditioned by fulfilling subsequent equation:

$$
T_{j, j \in A^{i}}^{i}>T_{S}^{i}
$$

To add the $C_{j}$ to $A^{i}$, all UEs currently served by the $C_{j}$ must still be experiencing higher capacity than capacity provided by the MBS.

The above description focuses on conditions and algorithm for inclusion of a cell into an active set. The opposite case, that is, removal of a cell from an active set, must be defined. In our proposal, the cell $C_{j}$ is deleted from the active set $A^{i}$ if its removal would result in lesser consumption of the MBS's radio resources. In other words, the cell $C_{j}$ is removed if condition (1) is no longer met. Of course, the cell is also removed if its backbone capacity or delay changes and do not fulfil either (2) or (3).

Among all cells included in $A^{i}$, a serving cell must be identified. Selection of serving cell is based on the comparison of signal levels measured by the UE. If the cell with the strongest signal measured by the $U E_{i}$ can fully served this particular $U E_{i}$, then the cell is selected as the serving cell. However, if this cell cannot provide enough capacity, then the cell providing maximum throughput is selected.

\section{System model for evaluation of active set management}

The setting and models of simulation performed in MATLAB follow the parameters presented in "System model, methodology, and simulation settings" section for evaluation of FCS performance in OFDMA networks with FAPs. This section describes especially parameters, in which both simulations differ.

Since the main objective is to assess when active set of the UEs should be updated, the outdoor UEs are supposed to be moving in comparison with the previous evaluations. The outdoor UEs are moving only outside the houses and they do not enter the building at all. A movement is generated according to probabilistic random waypoint mobility model [19] with a constant speed of $1 \mathrm{~m} / \mathrm{s}$. If an outdoor UE comes closer to a house, it just walks around it and does not go inside. On the other hand, indoor UEs stay fixed during the simulations. All UEs transmit data according to constant bit rate model with a bit rate in range from $60 \mathrm{kbps}$ to 4 Mbps for each UE. The bit rate is the same for all UEs in each simulation drop like in evaluation of OFDMA FCS in "Evaluation of FCS in ODFMA networks with femtocells" section. Nevertheless, each UE has different requirements on delay for its services. The required delay is selected among three possible classes: high demands (delay of backbone $\leq 50 \mathrm{~ms}$ ), medium demands (delay of backbone $\leq 75 \mathrm{~ms}$ ), and low demands (delay of backbone > $75 \mathrm{~ms}$ ). The selection of the delay requirements is done randomly. The probabilities that UE's demands on delay are high/medium/low equals to $5 / 20 / 75 \%$, respectively. The FAPs' backbone is limited to $8 \mathrm{Mbps}$ for downlink and $50 \%$ of its capacity is supposed to be occupied due to ADSL aggregation and signaling overhead. Consequently, the real available FAPs backbone capacity dedicated for the UEs in simulation is 4 Mbps. The capacity is shared by all UEs with equal priority since open access is considered. The delay profile of each backbone is determined according to delay measurement obtained from real network as presented in [20].

To eliminate the effect of random variables, the simulation duration is set to $1 \mathrm{~h}$ of real time and we run five simulation drops that are subsequently averaged out.

\section{Simulation results}

The results obtained by simulations are split into two sections. The first one represents the results for determination of appropriate $\alpha$ for the proposed algorithm. The second one shows the comparison of the proposed algorithm with competitive proposals.

\section{Evaluation of the proposed algorithm}

As shown in Figure 4, the frequency of active set update decreases with increase of $\alpha$ or with increase of offered traffic. This is due to limited capacity of the FAPs backbone. Once the FAPs backbone is fully utilized, the FAP is included into other active set(s) only if it improves throughput of the UE and ensures enough capacity even for all UEs with the FAP in current active set. Moreover, higher value of $\alpha$ lowers the active set update rate as well since higher profit must be achieved at the side of the MBS to include a FAP into the active set (see (1)). Active set update rate represents the average amount of changes

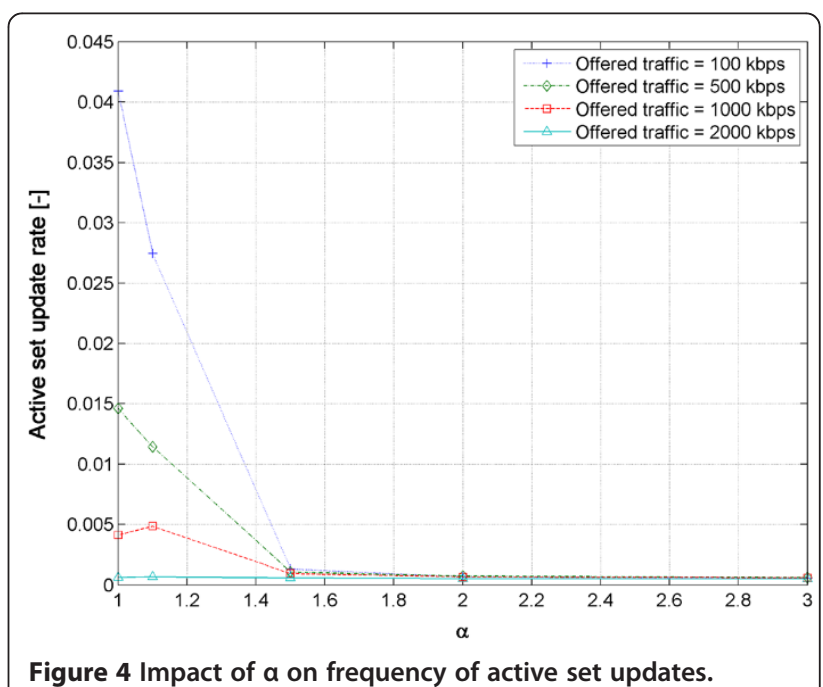


in active sets of all users per a simulation step (i.e., every second). In other words, the results achieved by indoor and outdoor UEs are averaged out. Each inclusion or removal of a cell into an active set represents one change.

Besides active set, $\alpha$ influences user's throughput as depicted in Figure 5 where the results are again averaged out for indoor and outdoor UEs. The throughput is affected by $\alpha$ only for high offered traffic loads. All cells, even FAPs, are able to serve high amount of users without reaching backbone limit for low offered traffic. Thus, no impact of $\alpha$ on throughput is observed. However, if offered traffic increases, then high $\alpha$ leads to selection of only considerably profitable cells as candidates to be included in the active sets. If $\alpha$ is low, each FAP is included in large number of active sets and all users connected to this FAP must share the limited backbone. Note that we assume proportional fair sharing of the FAPs backbone capacity among all UEs (indoor as well as outdoor) connected to it.

Based on the presented results, $\alpha>2$ is considered as appropriate gain since maximum throughput and minimum amount of active set updates are generated. For the purpose of evaluation of the proposal and competitive schemes, $\alpha=2$ and $\alpha=3$ are selected.

\section{Comparison of the proposed and competitive algorithms}

The proposal is compared with two other algorithms: conventional FCS active set management [7] and with the proposal on capacity-based FCS active set management proposed in [12]. Only these two proposals are considered since the selected proposal on capacity-based FCS outperforms any other similar proposals as presented in [12]. Conventional FCS is used only as a benchmark and we consider two levels of hysteresis: 3 $\mathrm{dB}$ (in figures denoted as $\Delta_{\mathrm{HM}}=3 \mathrm{~dB}$ ) and $5 \mathrm{~dB}$ $\left(\Delta_{\mathrm{HM}}=5 \mathrm{~dB}\right)$.

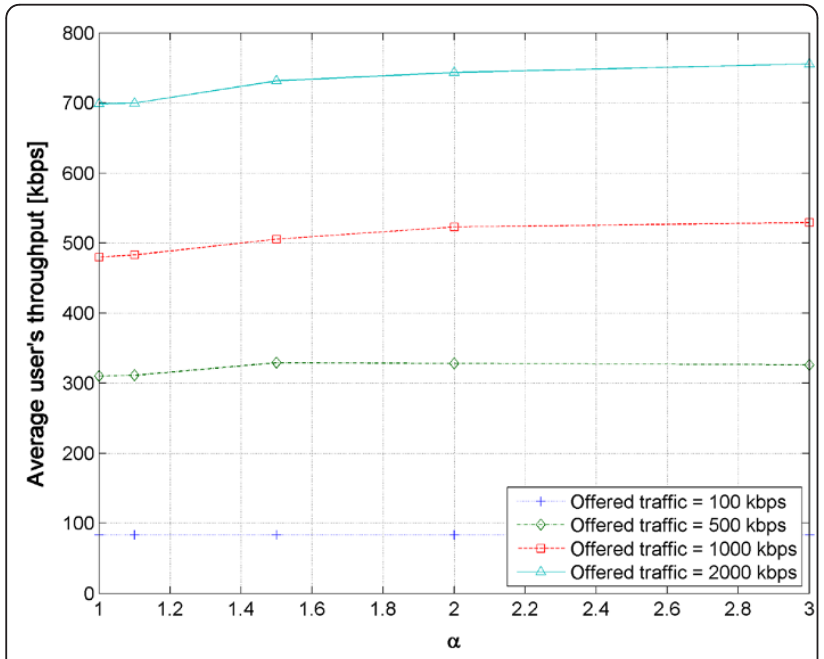

Figure 5 Impact of a on users throughput.
As shown in Figure 6, the proposed active set management algorithm improves throughput of all UEs (indoor as well as outdoor) comparing to the conventional and the capacity-based FCS (Figure 6c). The gain in throughput rises with the amount of offered data by the UEs and it is nearly independent on the value of $\alpha$ (excluding outdoor UEs with low offered traffic, where higher $\alpha$ leads to minor decrease in throughput). The throughput gain for indoor users (Figure 6a) is up to roughly 28, 17, and $41 \%$ comparing to capacity-based FCS, conventional FCS with 3 and $5 \mathrm{~dB}$ hysteresis, respectively. For outdoor users (Figure 6b), the proposed scheme and the capacity-based FCS perform in similar manner with only minor gain by the proposed algorithm. Comparing the proposal with the conventional FCS, the gain rises up to $20 \%$ with offered traffic up to $2000 \mathrm{kbps}$. For the offered traffic equal or higher than $2000 \mathrm{kbps}$, the gain gets stable and equals approximately to $7 \%$. The rapid drop experienced by the proposal and the capacity-based FCS at $2000 \mathrm{kbps}$ is due to the FAPs backbone limitation and it can be explained as follows. For each FAP, a UE is deployed indoor in our simulations. Therefore, this UE is attached to this FAP most of the time. Including the FAP in active sets of other outdoor UE, the backbone must be shared by all UEs with this FAP in the active set. Since available backbone capacity is $4000 \mathrm{kbps}$ in average in simulations, an inclusion of the FAP to an active set of any outdoor UE automatically limits the transmission capacity up to $2000 \mathrm{kbps}$.

Note that throughput of outdoor UEs is roughly ten times lower than that of indoor UEs. This is due to high number of active outdoor UEs deployed randomly among whole large simulation area sharing the MBS whereas only low number of UEs is connected to a FAP.

Frequency of active set updates is presented in Figure 7. Each event in active set update (either inclusion/deletion of a cell to/from active set) is linearly interconnected with certain amount of a management overhead. Therefore, these figures represent also the amount of control overhead generated due to active set management. In the case of indoor users only (Figure 7a), the lowest rate of active set update is reached by the conventional FCS. However, it is at the cost of significantly decreased throughput as presented in Figure 6a. The capacity-based FCS and the proposed scheme perform similarly in terms of active set update rate. The sudden rise in case of the proposal is again due to the backbone limit as explained above for throughput. Regarding outdoor UEs presented in Figure 7b, the results are exactly opposite. The lowest rate of active set update is reached by the proposal. The rate of updates decreases with higher values of $\alpha$ and with increase of offered throughput for our proposal. Lower frequency of active set updates for higher $\alpha$ and for high offered traffic is due to lower 


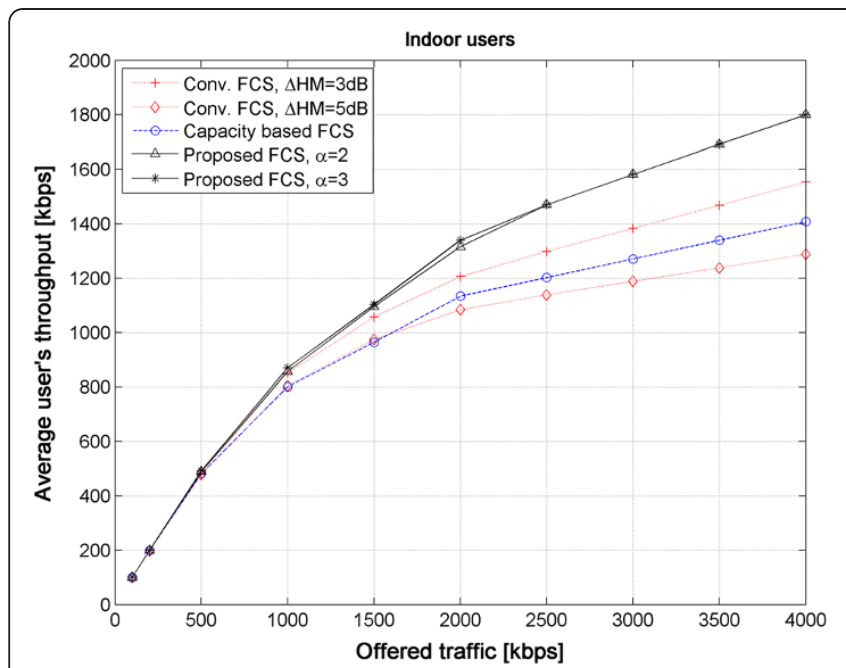

a)

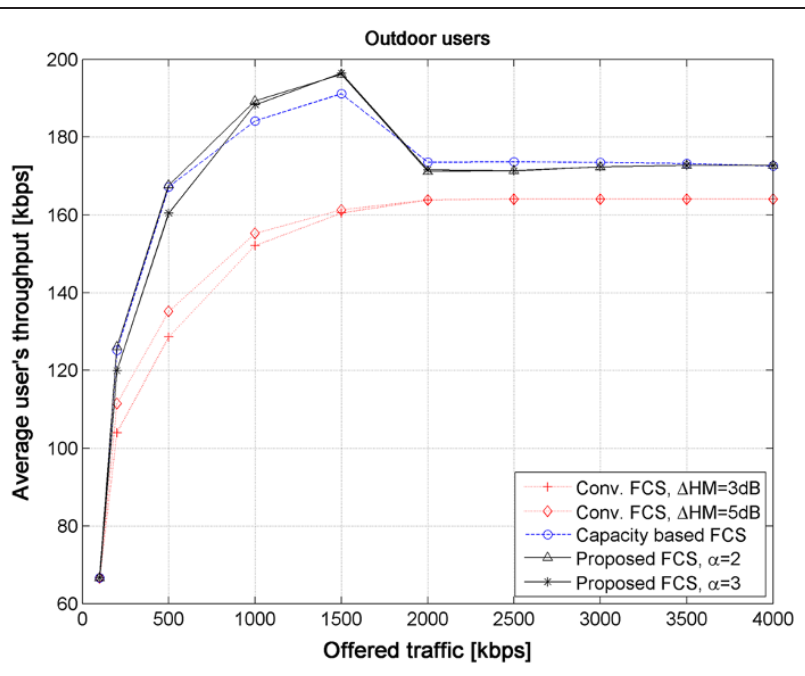

b)

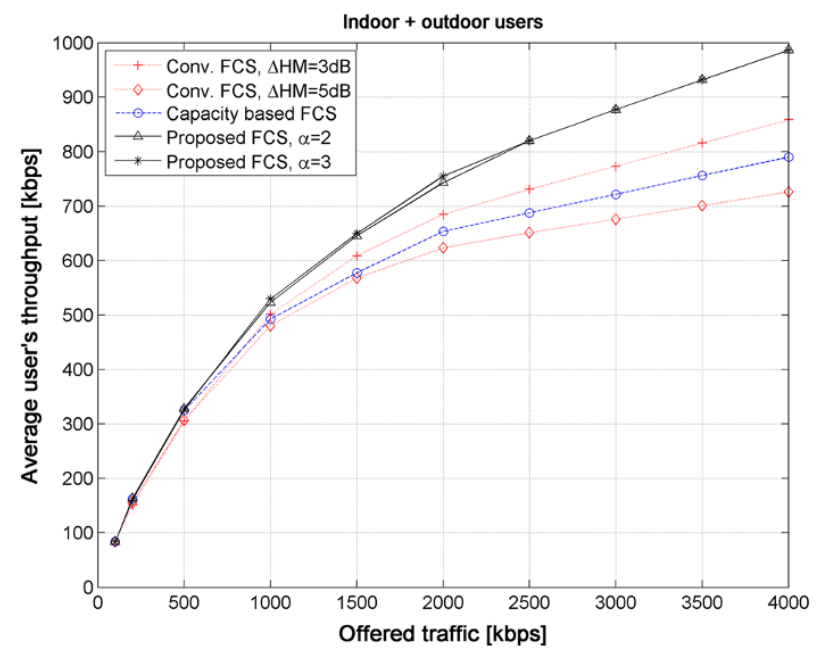

c)

Figure 6 Average throughput of UEs during simulation over amount of offered traffic by the UEs; throughput of: (a) only indoor users; (b) only outdoor users; (c) all users.

probability of fulfilling condition (1) and higher requirements on the FAPs backbone capacity, respectively. Note that frequency of active set updates of indoor users is roughly ten times lower comparing to outdoor users. Thus, the proposed scheme outperforms the conventional FCS and the capacity-based FCS roughly by $58-65$ and by $20-43 \%$ (depending on amount of offered traffic), respectively, if overall rate of active set updates (indoor as well as outdoor UEs) is compared (Figure 7c). Based on the results, we can state that the proposal generates significantly less overhead related to the active set management comparing to both competitive schemes.

Considering Figures 6 and 7, a marginal difference in throughput and active set update rate for outdoor UEs can be observed for both presented values of $\alpha$. While lower $\alpha$ slightly improves throughput of outdoor UEs, it is at the cost of minor rise in amount of active set updates.

In Figure 8, the average size of active set per a UE over whole simulation is depicted. For indoor UEs (Figure 8a), only roughly one cell is included in active set for the conventional FCS. This cell is typically a local FAP deployed in the same house as the UE. Other cells' signals (either FAPs' or MBSs') are usually attenuated significantly due to intervening walls. Hence, these cells do not provide signal with sufficient quality to be included in the active set. For the capacity-based FCS and the proposed FCS, roughly two cells are included in the active set on average. This is typically the MBS and the local FAP. Other FAPs provide weak signal (at least two walls are between the FAP and the UE) to be included in the active set. 


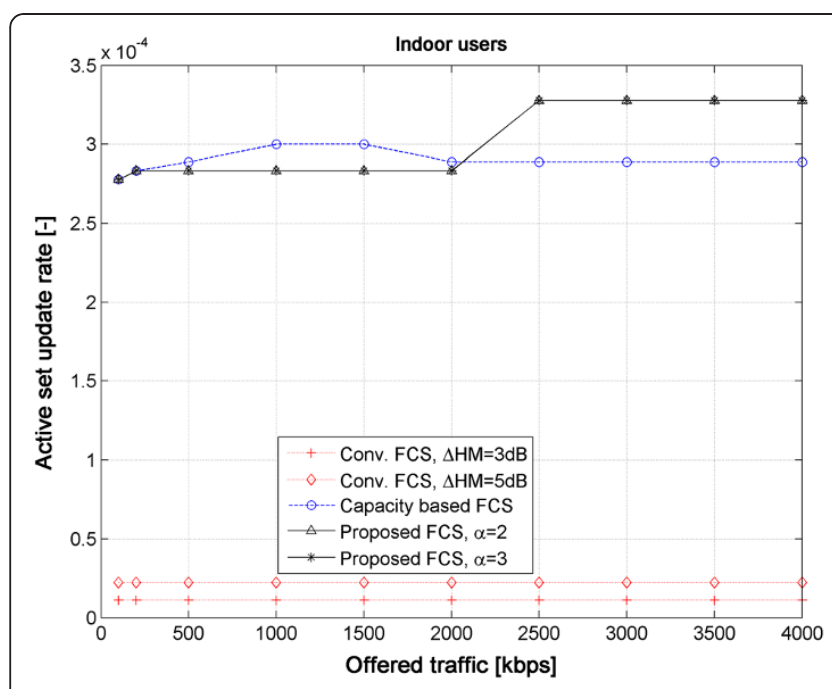

a)

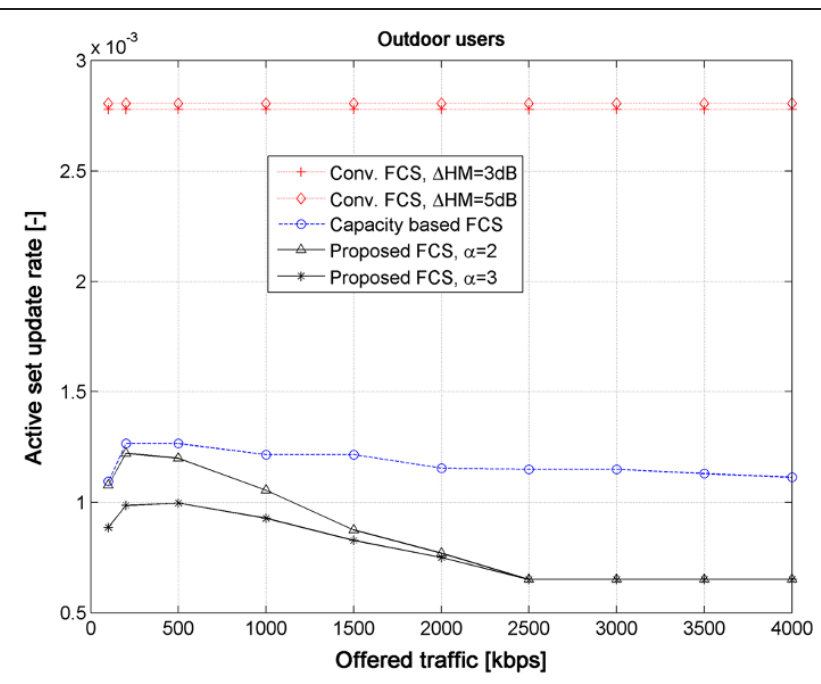

b)

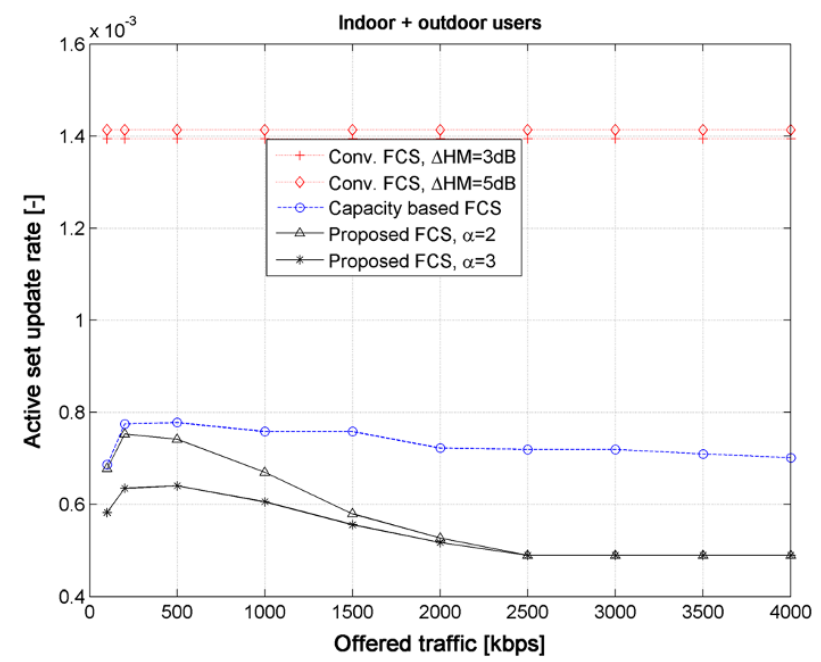

c)

Figure 7 Average amount of changes in active set of individual users per a simulation step; changes in active set of (a) only indoor users; (b) only outdoor users; (c) all users.

For outdoor users (Figure $8 \mathrm{~b}$ ), active set consists of the MBS and several closest FAPs. The exact number of the FAPs included in active set depends on offered traffic load for the capacity-based FCS. In the case of the proposal and the conventional FCS, the number of FAPs in active set is further influenced by $\alpha$ and by hysteresis level, respectively. The average size of active set is presented in Figure 8c. Note that based on standalone size of the active set, it can be concluded that neither lower nor higher active set size is profitable. This parameter just shows typical amount of cells involved in active set communication, which can be further used, for example, in optimization of cooperative communication.

Figure $9 \mathrm{a}-\mathrm{c}$ presents impact of individual FCS algorithms on the ratio of the time when user's requirements on capacity are not fulfilled. Comparing indoor (Figure 9a) and outdoor (Figure 9b) UEs, the satisfaction of outdoor UEs is lower. The reason is that indoor UEs are usually not limited by the radio capacity. The bottleneck is typically located on the FAP backbone, which is of higher capacity comparing to radio capacity of the MBS backbone.

The profit achieved by the proposal rises with offered traffic load and it is almost independent on value of $\alpha$ for indoor users. The improvement is up to 13,7 , and $12 \%$ when compared to the conventional FCS with $3-\mathrm{dB}$ hysteresis, with $5-\mathrm{dB}$ hysteresis, and the capacity-based FCS, respectively. For outdoor UEs, the maximum profit (50\%) is reached for $200 \mathrm{kbps}$ of the offered traffic if comparing the proposal with the conventional FCS. The 


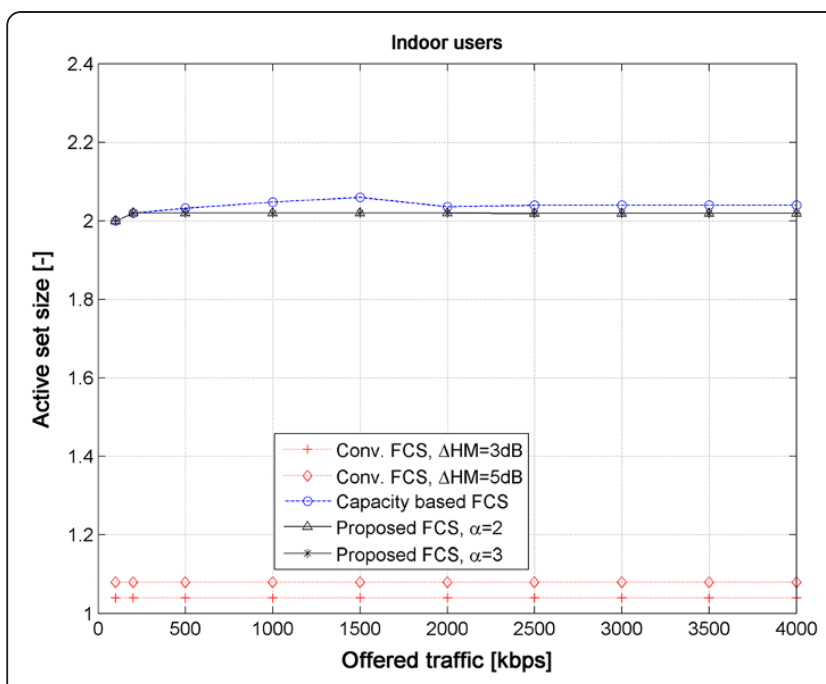

a)

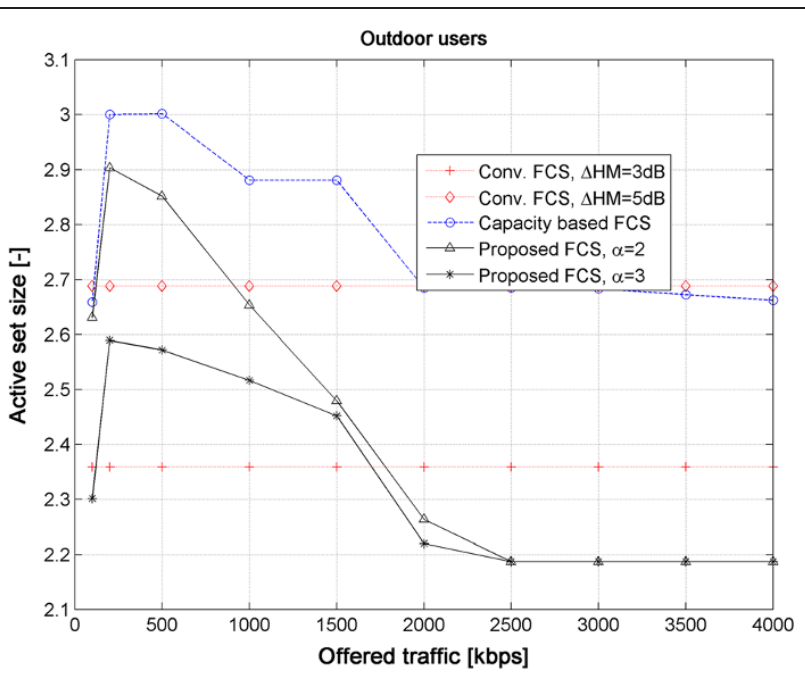

b)

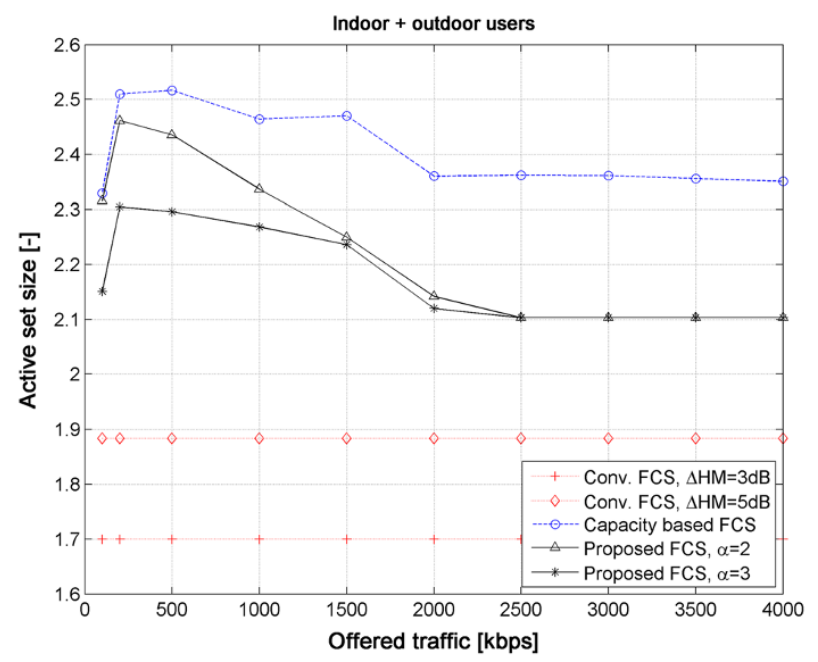

c)

Figure 8 Average amount of cells included in active set for (a) only indoor users; (b) only outdoor users; (c) all users.

performance of the capacity-based FCS and the proposed FCS is roughly the same for outdoor users. Nevertheless, the proposed algorithm is outperformed by none of both competitive algorithms for all UEs (indoor and outdoor) and for all offered traffic levels. The efficiency of the proposal comparing to both competitive schemes consists in more efficient selection of cells included in the active sets of individual UEs.

Capacity constraint for FAPs backbone also delay outage should be tackled. By delay outage is understood the situation when a UE's requirements on delay are not met. Our proposal suppresses the outage delay to the minimum achievable level. This minimum is given by occurrence of the situations when none of neighboring cell is able to provide sufficient delay. However, this problem is not related to FCS. The delay outage of competitive FCS schemes depends on the quality of FAPs backbone. During our simulations, the delay outage for both competitive FCS schemes was roughly in the range of $1-2.5 \%$ above the outage of our proposed scheme, which reaches delay outage under $1 \%$ even for heavy traffic load. Note that the delay outage introduced by our scheme is only due to overloading of the system by high amount of offered traffic by UEs.

\section{Control information for implementation of the proposed active set management}

To enable FCS in network with the FAPs, control information exchange among the FAPs and the MBSs must be defined. The information on backbone quality and FAP's radio quality must be reported. However, the quality of radio channel is periodically reported for conventional 


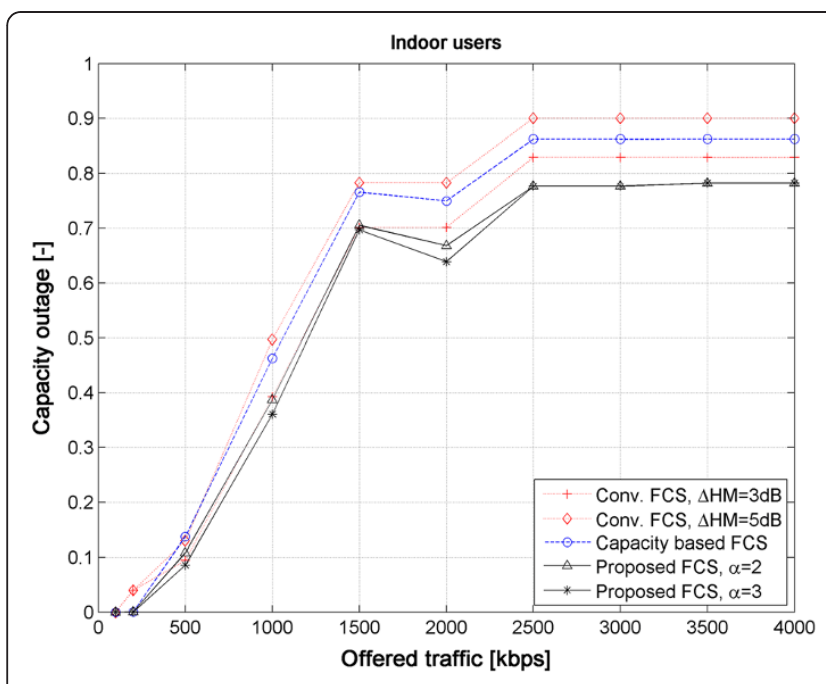

a)

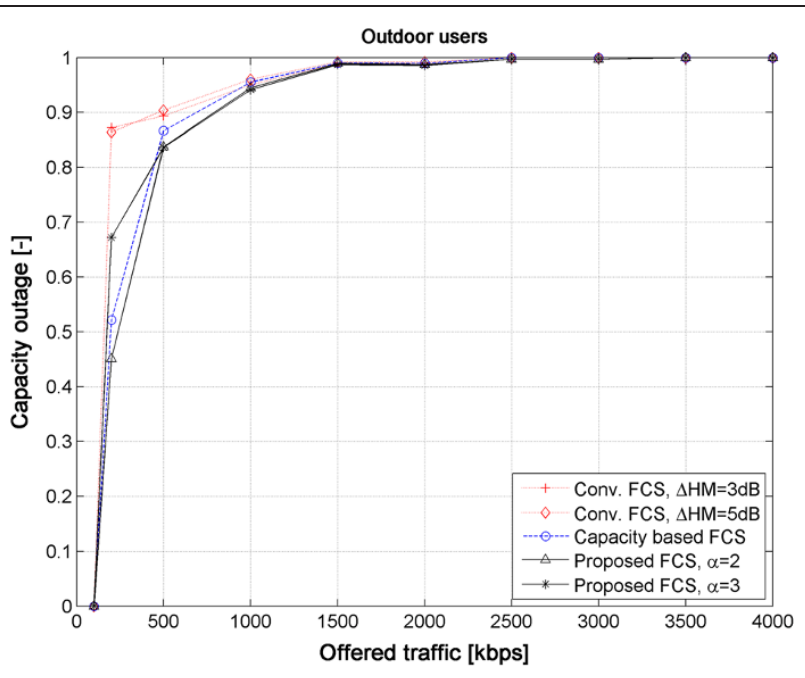

b)

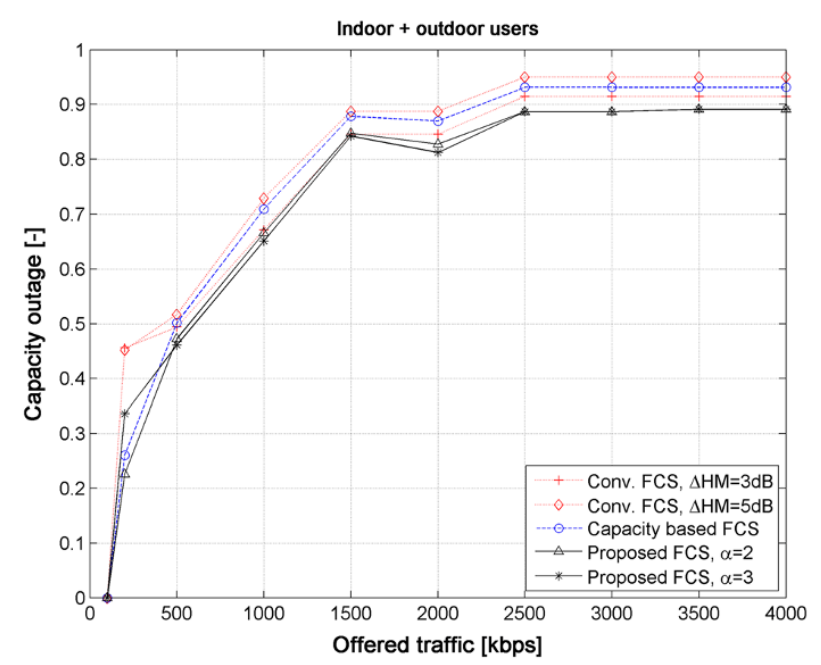

c)

Figure 9 Average ratio of time spent in the state when UEs requested capacity is not fully provided for (a) only indoor users; (b) only outdoor users; (c) all users.

handover purposes. Therefore, no additional overhead related to the radio channel quality is introduced by the proposed algorithm.

Each FAP is aware of its approximate backbone quality as it needs this information to schedule users' data over backbone. Moreover, estimation based on latest experienced backbone quality can be considered. Higher accuracy in derivation of the available backbone capacity is out of scope of this article and we let it for future research. Nevertheless, the information on backbone quality must be delivered to the serving cell, which is supposed to take control over the handover decision. Thus, the FAP should report available capacity and packet delay to the serving cell. The reporting of backbone quality can be included in control information for coordination of the MBSs and the FAPs.

The information on backbone delay can be provided in form of range of delays related to the experienced service class (e.g., voice, video streaming, FTP, etc.). It means that the delay does not need to be reported as an exact number, but only as an index representing appropriate range of delays. Therefore, its size is only of several bits. For example, 4 bits enable to distinguish 16 classes, which is sufficient number, higher than the amount of classes used by IP protocol or amount of classes defined for LTE-A. The information on capacity should be expressed as an absolute amount of available resources. The number of bits required for this information depends on accuracy of reporting information. 
Sufficient amount is 16 bits as it enables to distinguish $2^{16}$ levels of available capacity (for example., it corresponds to the resolution of $4 \mathrm{kbps}$ for $16 \mathrm{Mbps}$ backbone).

The transmission of information on backbone quality can be either triggered by a handover request or periodical. The drawback of the handover triggered reporting is additional delaying of handover (in tens of ms) due to delivering information on the backbone status to the MBS. However, its overhead is negligible since only few additional bits are transmitted per a handover. On the other hand, the periodic reporting does not delay handover but it increases overhead. The maximal amount of overhead in bps generated during the periodical reporting can be determined as

$$
B R_{\text {rep }}=\frac{S_{\text {ri }}}{T_{\text {rep }}}
$$

where $S_{\text {ri }}$ is the size of the reported information and $T_{\text {rep }}$ is the interval between two reports (in seconds). The maximum size of a report is $16+4$ bits as stated earlier. The minimum reporting period is supposed to be equal to the frame duration, which is $10 \mathrm{~ms}$ in LTE-A. Then the maximum reporting overhead is $2 \mathrm{kbps}$. This amount of overhead is still negligible comparing to the expected backbone capacity in Mbps.

Like in the conventional FCS as introduced in "FCS in OFDMA networks with femtocells" section or in the capacity-based FCS, information on status of radio resources (muted or not) must be forwarded by the serving cell to all cells in the active set. This information is carried in FCS command (see Table 1). Note that the information on occupied resources is delivered to the cells in active set even in the conventional FCS. Therefore, the only difference in overhead is in delivery of information on muting. The information on muting for each cell in active set is represented by 1 bit (just on or off is indicated). Considering average size of the active set around two cells (see Figure 8), the overhead due to our proposal is up to $0.2 \mathrm{kbps}$ (two cells reported once per 10 $\mathrm{ms}$ frame). Even if this message introduces additional overhead, the overhead is increased only negligibly related to the conventional FCS (difference of tens of bps). Contrary, the overhead is even slightly lower (again only tens of bps) than in case of the capacity-based FCS since the active set size of our proposal is lower (see Figure 8).

\section{Conclusions}

The problems related to adaptation of CDMA FCS to fits OFDMA networks with femtocells are described in this article and appropriate solutions are proposed as well. The evaluation confirms the improvement of the network performance by using FCS for outdoor as well as for indoor users. It also shows good compatibility between FCS and opportunistic scheduling techniques, as FCS brings significant improvement in this context as well. To further enhance gain introduced by FCS, novel algorithm for management of active set for FCS is proposed. The algorithm considers backbone limitations introduced by deployment of the FAPs in networks. The proposed algorithm is based on comparison of amount of the MBS's radio resources consumed if a cell is included to the UE's active set or not. Notable increase in throughput for indoor as well as for outdoor users is demonstrated by the simulations. Simultaneously, the amount of generated overhead is significantly reduced by the proposal. Moreover, the proposed algorithm reduces the time when users are not fully satisfied with experienced capacity and delay.

As the simulations show, the most efficient active set always contains an MBS. For indoor users, the closest FAP deployed in the same house should be included as well. The amount of FAPs included in active set together with the MBS for outdoor users depends on mutual distance between the UE and neighboring FAPs. Further, the number of FAPs slightly varies with offered traffic level. In average, roughly 1.3 FAPs and 1.5 FAPs are included in active set of an outdoor UE if this UE offers low (less than $100 \mathrm{kbps}$ ) or high (above $1500 \mathrm{kbps}$ ) traffic and medium traffic (100-1500 kbps), respectively.

The proposed algorithm can be further extended for FAP's downlink power control to reduce interference from cells that cannot fulfil UE's requirements.

\section{Competing interests}

'The authors declare that they have no competing interest.

\section{Acknowledgment}

This work has been performed in the framework of the FP7 project FREEDOM IST-248891 STP, which is funded by the European Community. The authors would like to acknowledge the contributions of their colleagues from FREEDOM Consortium (http://www.ict-freedom.eu).

\section{Author details}

'Department of Telecommunication Engineering, Czech Technical University in Prague, Technicka 2, 16627, Prague, Czech Republic. ${ }^{2}$ CEA, LIST, Communicating Systems Laboratory, Centre de Saclay, Point Courrier 173, 91191, Gif Sur Yvette Cedex, France.

Received: 21 November 2011 Accepted: 24 August 2012

Published: 18 September 2012

\section{References}

1. ITU-R Document 5D/TEMP/89r1, Draft new Report ITU-R M.[IMT.TECH], Requirements related to technical system performance for IMT-Advanced radio interface(s) (2008)

2. M.Z. Chowdhury, W. Ryu, E. Rhee, Y.M. Jang, Handover between macrocell and femtocell for UMTS based networks, in 11th International Conference on Advanced Communication Technology ICACT 2009, ed. by (Phoenix Park, Korea, 2009). 15-18 February

3. G. de la Roche, A. Valcarce, D. Lopez-Perez, J. Zhang, Access control mechanisms for femtocells. IEEE Commun Mag 48(1), 33-39 (2010). doi:10.1109/MCOM.2010.5394027 
4. T. Jansen, I. Balan, J. Turk, I. Moerman, T. Kurner, Handover parameter optimization in LTE self-organizing networks, in IEEE VTC 2010-Fall, ed. by (Ottawa, Canada, 2010). 6-9 September

5. M. Kamoun, L. Mazet, Base-station selection in cooperative single frequency cellular network, in IEEE Signal Processing Advances in Wireless Communications SPAWC 2007, ed. by (Helsinki, Finland, 2007). June 17-20

6. 3GPP TR 25.433 v10.4.0, Technical Specification Group Radio Access Network; UTRAN lub interface Node B Application Part (NBAP) signalling (2011). Release 10, 27 September

7. 3GPP TR 25.848 v4.0.0, Technical Specification Group Radio Access Network: Physical layer aspects of UTRA High Speed Downlink Packet Access, Technical report (2001). Release 4, March

8. S.-W. Kim, Y.-H. Lee, Adaptive MIMO mode and fast cell selection with interference avoidance in multi-cell environments, in Fifth International Conference on Wireless and Mobile Communications ICWMC '09, ed. by (Cannes, France, 2009). 23-29 August

9. H.H. Choi, J.B. Lim, H. Hwang, K. Jang, Optimal handover decision algorithm for throughput enhancement in cooperative cellular networks, in IEEE VTC 2010-Fall, ed. by (Ottawa, Canada, 2010). 6-9 September

10. H.H. Choi, An optimal handover decision for throughput enhancement. IEEE Commun Lett 14(9), 851-853 (2010). doi:10.1109/ LCOMM.2010.09.092356

11. NTT DOCOMO, Investigation on Coordinated Multipoint Transmission Schemes in LTE-Advanced Downlink, in 3GPP TSG RAN WG1 Meeting \#55bis, R1-060298, ed. by (Ljubljana, Slovenia, 2009). 12-16 January

12. M. Feng, X. She, L Chen, Y. Kishiyama, Enhanced dynamic cell selection with muting scheme for DL COMP in LTE-A, in IEEE VTC 2010-Spring, ed. by (Taipei, Taiwan, 2010). 16-19 May

13. K.-W. Lee, J.-Y. Ko, Y.-H. Lee, K.-W. Lee, J.-Y. Ko, Y.-H. Lee, Fast cell site selection with interference avoidance in packet based OFDM cellular systems, in IEEE Globecom 2006, ed. by (San Francisco, USA, 2006). 27 November-1 December

14. A. Das, B. Krishna, F. Khan, S. Ashwin, H.-J. Su, Network controlled cell selection for the high speed downlink packet access in UMTS, in IEEE WCNC 2004, ed. by (Atlanta, USA, 2004). 21-25 March

15. H. Fu, D. Kim, Scheduling performance in downlink WCDMA networks with AMC and fast cell selection. IEEE Trans Wirel Commun 7(7), 2580-2591 (2008). doi:10.1109/TWC.2008.060956

16. FemtoForum, Interference management in OFDMA Femtocells (2010). February

17. ITU Recommendation ITU-R M.1225, Guidelines for evaluation of radio transmission technologies for IMT 2000 (Guidelines for evaluation of radio transmission technologies for IMT 2000, Guidelines for evaluation of radio transmission technologies for IMT 2000) (1997)

18. C. Yu, W. Xiangming, L. Xinqi, Z. Wei, Research on the modulation and coding scheme in LTE TDD wireless network, in International Conference on Industrial Mechatronics and Automation ICIMA 2009, ed. by (Chengdu, 2009)

19. T. Camp, J. Boleng, V. Davies, A survey of mobility models for ad hoc network research. Wirel Commun Mob Comput 2(5), 483-502 (2002). Special issue on Mobile Ad Hoc Networking: Research. Trends and Applications

20. H. Hariyanto, T. Nugraha, A. Saufi, J. Stephan, Y. Corre, Trial report, Deliverable D6.2 of ICT-248891 STP FREEDOM project (2011)

\section{Submit your manuscript to a SpringerOpen ${ }^{\circ}$ journal and benefit from:}

- Convenient online submission

- Rigorous peer review

- Immediate publication on acceptance

- Open access: articles freely available online

- High visibility within the field

- Retaining the copyright to your article

Submit your next manuscript at $\gg$ springeropen.com 